\title{
The Influence Effect of Coupling on Solid-Phase Conversion in the Plate from Composition Materials under Condition of Axial Tension and Shifting
}

\author{
Yurii A. Chumakov ${ }^{1, \text { a) }}$ and Anna G. Knyazeva ${ }^{1,2, b)}$ \\ ${ }^{1}$ Institute of Strength Physics and Materials Science SB RAS, Tomsk, 634055, Russia \\ ${ }^{2}$ National Research Tomsk Polytechnic University, Tomsk, 634050, Russia \\ a) Corresponding author: chya@ispms.tsc.ru \\ b)anna@ispms.tsc.ru
}

\begin{abstract}
The paper considers the influence of the heating character and type of kinetic function on stress-deformed state of plate with solid-phase chemical reaction. The strained sate was caused by uniaxial tension, impulse heating and chemical reaction. The planar stressed state approach was employed. The influence of the thermal and mechanical coupling processes on temperature, stress and deforms fields under uniaxial tension condition was investigated. The influence of formal kinetic laws on macroscopic characteristics of process was shown.
\end{abstract}

Keywords: mechanical stresses, impulse heating, coupled processes, uniaxial tension, mathematical model

\section{INTRODUCTION}

The influence of mechanical stresses on the rate of chemical reactions in condensed phases has been studied by researchers for many decades [1-3]. The mathematical modeling of similar phenomena considers the features solidphase reactions (heterogeneous character of the reactions, the important role of transport processes and the interdependence of the different physical phenomena) [4-7]. But the coupled processes of different physical nature are not included in many models. For example, Chumakov and Knyazeva [8] considered the influence of some physical fields on other, Dickinson and Heal studied the variation of a kinetic law forms [9]. Authors [10] investigated the different coupled processes by the way of calculation of effective physical properties.

The present work is devoted to the influence of the stressed-deformed state on propagation regimes of solid phase exothermic chemical reaction with based on coupling of deformation, temperature and concentration fields.

\section{MATHEMATICAL FORMULATION}

The fixed plate of the reactive substance under external uniaxial tension is considered. Authors assume that the length of the plate $L_{x}$, width $L_{y}$, height $L_{z}$ and the conditions $L_{z} \ll L_{x}, L_{z} \ll L_{y}$ are satisfied. The coordinate axes are shown in Fig. 1. The chemical reaction propagates along the $X$-axis. The external loading direction is perpendicular to that of chemical reaction propagation. Authors consider the melting point is not reached and the chemical reaction occurs in solid phase. The exothermal reaction is assumed to describe summary schema $A \rightarrow B$. Authors vary the form of rate reaction kinetic function and type of heating source in this work.

To estimate stressed-deformed state of plate and influence of coupling field on solid-phase reaction we consider the uniaxial tension problem (with chemical reaction) which consists of two parts [11]. These parts are independently solved.

International Conference on Physical Mesomechanics of Multilevel Systems 2014

AIP Conf. Proc. 1623, 95-98 (2014); doi: 10.1063/1.4898891

(C) 2014 AIP Publishing LLC 978-0-7354-1260-6/\$30.00 


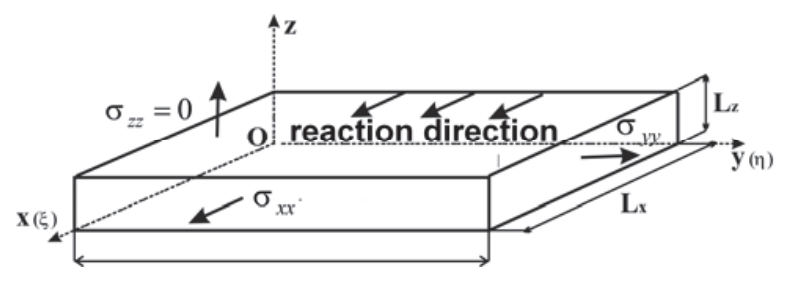

FIGURE 1. Illustration of the formulation of the problem

The first part of the problem consists of the heat equations and the reaction kinetics equation and the boundary conditions and it describes the propagation of solid-phase chemical reaction

$$
\begin{gathered}
c \rho \frac{\partial T}{\partial t}=\lambda_{T}\left(\frac{\partial^{2} T}{\partial x^{2}}+\frac{\partial^{2} T}{\partial y^{2}}\right)+Q \varphi(\eta, T)-3 K T \alpha_{T} \frac{\partial \varepsilon_{k k}}{\partial t}, \\
\frac{\partial \eta}{\partial t}=k_{0} \varphi_{1}(\eta) \varphi_{2}(T), \\
t=0: T=T_{0}, \eta=\eta_{0}, x=0:-\lambda \frac{\partial T}{\partial x}=q(t), \quad x=L_{x}: \frac{\partial T}{\partial x}=0, \quad y=0: \frac{\partial T}{\partial y}=0, \quad y=L_{y}: \frac{\partial T}{\partial y}=0,
\end{gathered}
$$

where $c, \rho$ and $\lambda_{T}$ are the heat capacity, density and thermal conductivity, $T$ is the plate temperature, $\eta$ is the degree of conversation or the fraction of the reaction product, $Q$ is the heat effect of the overall reaction, $\varphi_{1}(\eta)$ is the kinetic function, $\varphi_{2}(T)=\exp \left(-E_{a} /(R T)\right)$ is the temperature dependence of reaction rates, $x$ and $y$ are coordinates, $\varepsilon_{k k}=\varepsilon_{x x}+\varepsilon_{y y}+\varepsilon_{z z}$ is the first invariant of the strain tensor, $\alpha_{T}$ - thermal expansion coefficient, $K$ is the isothermal bulk modulus.

Authors consider heat source is the impulse source can be represented as:

$$
q(t)=q_{0} f(t), f(t)=\sum_{k=1}^{n} f_{k}(t), f_{k}(t)=\left\{\begin{array}{l}
1,\left(t_{i}+t_{p}\right)(k-1) \leq t<t_{i}+\left(t_{i}+t_{p}\right)(k-1), \\
0, t_{i}+\left(t_{i}+t_{p}\right)(k-1) \leq t<\left(t_{i}+t_{p}\right) k,
\end{array}\right.
$$

where $t_{i}, t_{p}$ are radiation duration and pause, $n$ is the number of pulses, $q_{0}$ is the maximum power density of the external heat source (heat flux density), $r$ is the beam radius.

The second part of general problem is the mechanical equilibrium problem which consists of two equilibrium equation

$$
\frac{\partial \sigma_{x x}}{\partial x}+\frac{\partial \sigma_{x y}}{\partial y}=0, \frac{\partial \sigma_{x y}}{\partial x}+\frac{\partial \sigma_{y y}}{\partial y}=0 .
$$

The components of strain tensor are related to that of stress tensor using Duhamel-Neumann relations and can be represented as:

$$
\sigma_{x x}=\frac{E}{1-\mu^{2}}\left[\varepsilon_{x x}+\mu \varepsilon_{y y}-\frac{1+\mu}{3} \omega\right], \quad \sigma_{y y}=\frac{E}{1-\mu^{2}}\left[\varepsilon_{y y}+\mu \varepsilon_{x x}-\frac{1+\mu}{3} \omega\right], \quad \sigma_{x y}=\frac{E}{2(1+\mu)} \varepsilon_{x y},
$$

where $\omega=3\left[\alpha_{T}\left(T-T_{0}\right)+\left(\alpha_{p}-\alpha_{r}\right) \eta\right]$ is the volumetric expansion function, $\left(\alpha_{\mathrm{p}}-\alpha_{\mathrm{r}}\right)$ is the volume changes due to the discrepancy between the final and initial phases, $\mu$ is Poisson's ratio, $E$ is the elastic modulus.

Differentiated the first equation (4) with respect to $x$ and the second equation (4) with respect to $y$, we get

$$
\frac{\partial^{2} \sigma_{x y}}{\partial x \partial y}=-\frac{\partial^{2} \sigma_{x x}}{\partial x^{2}}, \frac{\partial^{2} \sigma_{x y}}{\partial x \partial y}=-\frac{\partial^{2} \sigma_{y y}}{\partial y^{2}} .
$$

Using equations (4), (5) and the strain compatibility conditions [12], we find 


$$
\Delta \sigma_{k k}=-\frac{E}{3} \Delta \omega,
$$

where $\sigma_{k k}=\sigma_{x x}+\sigma_{y y}$. Subtracting the second equation (6) of the first and adding to both sides of the $\partial^{2} \sigma_{y y} / \partial x^{2}$, can be obtained

$$
\Delta \sigma_{y y}=\frac{\partial^{2} \sigma_{k k}}{\partial x^{2}} .
$$

Differentiating the first equation (4) with respect to y and the second equation (4) with respect to $x$, can be found

$$
\Delta \sigma_{x y}=-\frac{\partial^{2} \sigma_{k k}}{\partial x \partial y} .
$$

It was assumed that the uniform load $P$ along the $y$ axis was applied to the ends of the plate at the $x=0$ and $x=L_{x}\left(\sigma_{y y}=P\right)$. The boundaries of the plate are free at the $y=0$ and $y=L_{y}$.

As a result, the stress tensor components can be determined using the equations (7)-(9) with boundary conditions

$$
x=0: x=L_{x}: \sigma_{x x}=0, \sigma_{y y}=P, \sigma_{x y}=0, y=0, y=L_{y}: \sigma_{x x}=0, \sigma_{y y}=P, \sigma_{x y}=0 .
$$

Other type of loading is simple shear. In this case, two loads were applied to the ends of plate $\sigma_{x x}=P_{1}$ at the $x=0$ and $x=L_{x}$ and $\sigma_{y y}=P_{2}$ at the $y=0$ and $y=L_{y}$. The result of these loads is directed along the diagonal line of the plate. This condition is executed in the elasticity theory problems if $P_{1}=P \cos \alpha, P_{1}=P \sin \alpha$, where $\operatorname{tg} \alpha=L_{x} / L_{y}$. So, other the boundary conditions can be presented as:

$$
x=0, x=L_{x}: \sigma_{x x}=P \cos \alpha, \sigma_{y y}=0, \sigma_{x y}=0, y=0, y=L_{y}: \sigma_{x x}=0, \sigma_{y y}=P \sin \alpha, \sigma_{x y}=0 .
$$

The strains tensor components can be found using (5). The influence of type of the kinetic function $\varphi_{1}(\eta)$ on stressed-deformed state of plate is studied in the present work.

The problem was solved numerically. The successive over relaxation method was used to solve the mechanical equilibrium problem. The differential equations included into system (1)-(3) were approximated by difference equations; the resulting system of linear algebraic equations was solved by a sweep method. In calculations, the temperature, concentration, stress and strains distributions in the plate at different time instants by varying the physical parameters were determined.

It is considered that the plate is ceramic based on $\mathrm{TiC}$. All the calculations presented below were carried out with the following values of the parameters [13]: $\lambda=21.9 \mathrm{~W} /(\mathrm{m} \times \mathrm{K}), \rho=4900 \mathrm{~kg} / \mathrm{m}^{3}, c=692 \mathrm{~J} /(\mathrm{kg} \times \mathrm{K}), E_{\mathrm{a}}=160 \mathrm{~kJ} / \mathrm{mol}$, $R_{\mathrm{b}}=8.31 \mathrm{~J} /(\mathrm{mol} \times \mathrm{K}), Q=7 \times 10^{9} \mathrm{~J} / \mathrm{kg}, L_{x}=L_{y}=0.01 \mathrm{~m}, \alpha_{T}=8 \times 10^{-6} \mathrm{~K}, \alpha_{\mathrm{C}}=10^{-7}, v=0.33, E=490 \mathrm{GPa}$.

\section{RESULTS AND DISCUSSION}

The three variants of coupled models are considered:

1. The chemical reactions are absent, i.e. $\varphi_{1}(\eta)=0$.

2. The chemical reaction rate depends on temperature by the Arrhenius law and it depends on the degree of conversation as $\varphi_{1}(\eta)=1-\eta$.

3. The inhibition of the reaction products is taken into account in the kinetic function as $\varphi_{1}(\eta)=\exp \left(-m_{0} \eta\right) \eta^{-n_{0}}$, where $m_{0}, n_{0}$ are the inhibition parameters.

Figure 2 shows the special distribution of temperature, the first invariant of the stress tensor at the moment $t=$ 0.015 and maximum temperature of plate. As can be observed, the impulse character of heating plate (Fig. 2(a)): at first, the maximum temperature increases smoothly until the end of the impulse $t_{i}$, then the temperature decrease during time, i.e. until $t_{\mathrm{p}}$, next impulse leads to further heating (curve 1, Fig. 2(a)) or the heat release due to active chemical conversion is increased (curves 2 and 3, Fig. 2(a)). The inhibition of the reaction product layer results in slower increasing of temperature due to the chemical reaction (curve 3, Fig. 2(a,c)).

The assumption of coupled of the thermal and deformation fields leads to arising of non-unidimensionality border effect. The temperature profile has a "bend" due to mechanical dissipation and interaction of thermal and mechanical processes (Fig. 2(b)). If only thermal stresses are considered, the maximum mechanic stresses reach a value of $1.5 \mathrm{GPa}$ (curves 2, 3, Fig. 2(d)). The concentration expansion of reaction products leads to increasing of stress, that of magnitude becomes comparable to the applied external load (curve 2, Fig. 2(d)). 

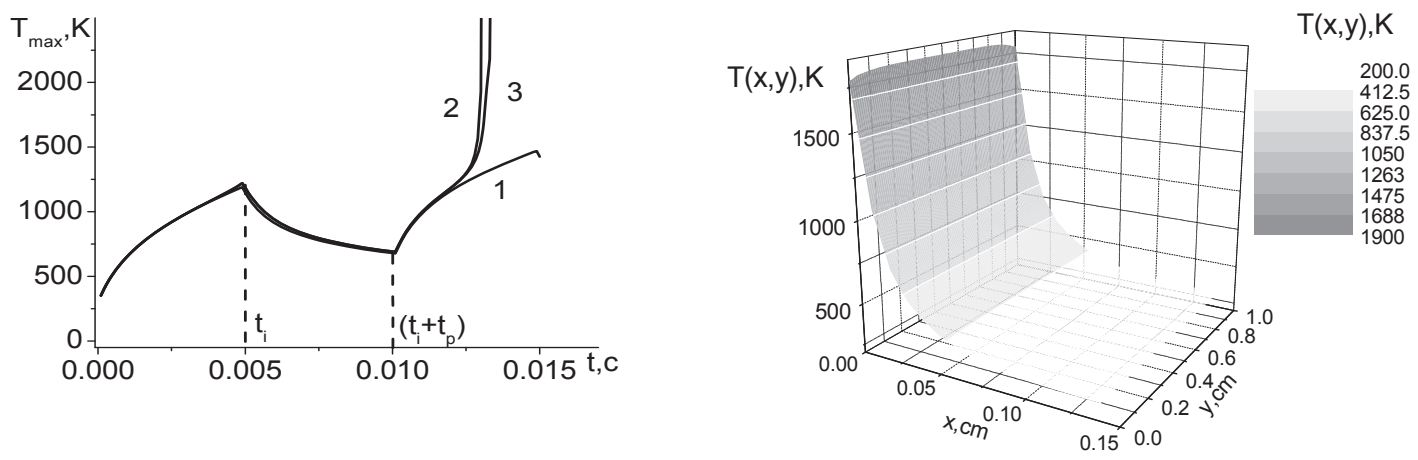

(a)
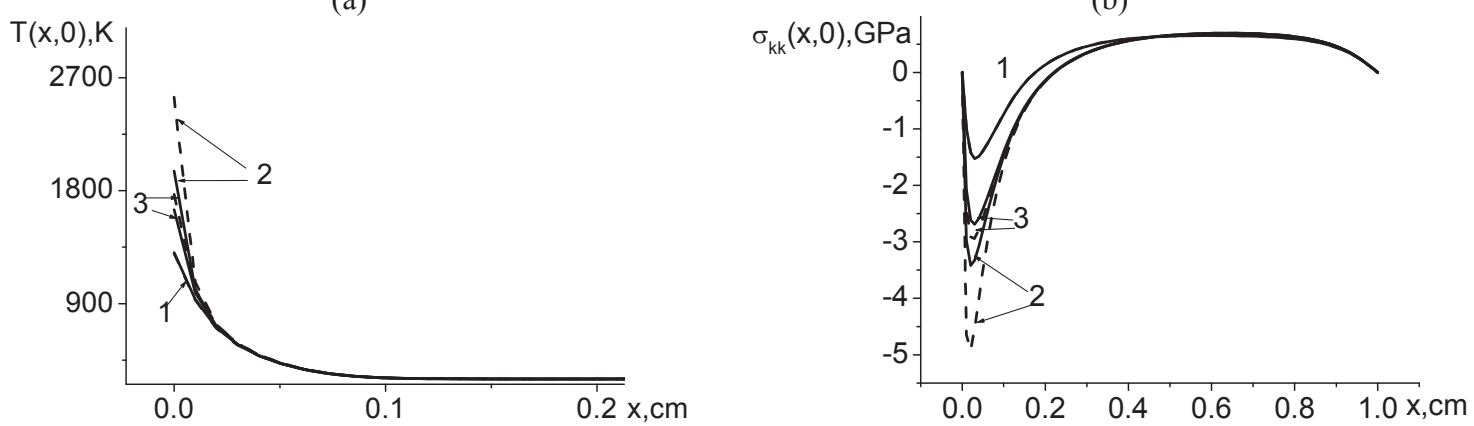

(c)

(d)

FIGURE 2. Maximum temperature of plate (a) and spatial distributions of the plate temperature (b), the temperature (c) and the first invariant of the stress tensor (d) along $x$-axis at the moment $t=0.015 \mathrm{~s}: t_{\mathrm{i}}=t_{\mathrm{p}}=5 \times 10^{-3} \mathrm{~s}, n=10, q_{0}=10^{7} \mathrm{~W} / \mathrm{m}^{2}$, $P=1 \mathrm{GPa}$. The chemical reactions are absent (1); the first-order reaction (2); the inhibition of the reaction products is taken into account in the kinetic function $\left(m_{0}=2, n_{0}=0\right)(3)$

\section{CONCLUSIONS}

A model of uniaxial tension plate under impulse heating with allowance for interaction of thermal and mechanical processes, as well as the concentration expansion was proposed in the paper. As shown in the example of $\mathrm{TiC}$ system, the thermal and mechanical coupling processes lead to non-unidimensionality border effects and decrease of the maximum temperature. Also it was shown that taking into account the coupling of thermal, concentration and mechanical fields, the internal stresses caused by chemical reaction can reach the values comparable to the applied external load.

\section{REFERENCES}

1. P. Yu. Butyagin, Russ. Chem. Rev. 63(12), 965 (1994).

2. N. S. Enikolopyan, V. B. Voleva, and A. A. Hzardzhyan, Dokl. Akad. Nauk USSR 292(5), 1165 (1987).

3. N. S. Enikolopyan, Zhurn. Fiz. Khim. 63(9), 2289 (1989).

4. V. K. Smolyakov, Combust. Explos. Shock 25(5), 582 (1989).

5. V. P. Radchenko, P. I. Krasnoschekov, and A. F. Fedotov, Bull. SamSTU. Phys. Math. Sci. 26, 102 (2004).

6. P. I. Krasnoschekov and A. F. Fedotov, Bull. SamSTU. Phys. Math. Sci. 43, 81 (2006).

7. V. A. Belyaeva, Bull. Udmurt Univer. Mech. 2, 102 (2008).

8. Yu. A. Chumakov and A. G. Knyazeva, Fiz. Mezomekh. 16(2), 85 (2013).

9. C. F. Dickinson and G. R. Heal, Thermochim. Acta 340-341, 89 (1999).

10. N. N. Nazarenko and A. G. Knyazeva, Fiz. Mezomekh. 14(5), 71 (2011).

11. N. K. Evstigneev and A. G. Knyazeva, Comb. Expl. Shock Waves 46(3), 307 (2010).

12. L. I. Sedov (Nauka, Moscow, 1995).

13. A. P. Babichev, N. A. Babushkina, and A. M. Bratkovskii (Energatomizdat, Moscow, 1991). 